\title{
A Comparison of aggregation behavior in aqueous humic acids
}

\author{
Christi Young and Ray von Wandruszka* \\ Department of Chemistry, University of Idaho, Moscow ID 83844-2343, USA. \\ E-mail:rvw@uidaho.edu
}

Received 3rd January 2001, Accepted 29th January 2001 Published on the web 13th February 2001

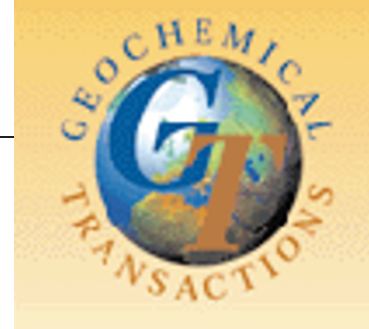

Published on the web 13th February 2001

\section{Article}

The ability of six humic acids (HAs) to form pseudomicellar structures in aqueous solution was evaluated by five techniques: size exclusion chromatography; pyrene fluorescence enhancement; the pyrene $I_{1} / I_{3}$ ratio; the cloud point of dilute HA solutions; and the fluorescence anisotropy of HAs. Soil HAs were found to aggregate most easily, both on microscopic and macroscopic scales. The formation of amphiphilic structures was chiefly related to HA-solvent interactions: highly solvated HAs aggregated poorly, while a lignite derived material underwent intermolecular, rather than intramolecular, rearrangements. A newly discovered algal HA was found to have minimal aggregative properties.

\section{Introduction}

Humic substances are the breakdown products of plant and animal matter in environmental matrices, especially soil. The major components of these materials - humic acid (HA), fulvic acid (FA), and humin - are defined on the basis of their solubilities in aqueous solution. HA, the subject of the present study, is soluble in solutions of $\mathrm{pH}>2$, while FA is soluble at all pHs and humin is entirely insoluble. HA is comprised of a suite of polydisperse carbonaceous polymers with molecular weights ranging to tens of $\mathrm{kDa}^{1}{ }^{1}$ Both molecular size and functional group characteristics are variable, depending on the origin of the material and the environmental factors implicated in its formation. Considerable diversity also exists within a single HA sample. Generally, however, HAs have a significant aromatic content (30-60\% of C) and an abundance of carboxy $\left(15-25 \%\right.$ of C) and hydroxy groups. ${ }^{2}$ Their behavior in aqueous solution has been studied extensively ${ }^{3-6}$ and it has been noted that they have significant surface activity.

Based on this observation, the detergent model of HA was developed $^{7-10}$ and has been found to account for much of its behavior in aqueous solution. According to this model, HA polymers aggregate spontaneously in water, forming micellelike structures comparable to those formed by synthetic surfactants. The term "pseudomicelle" is often used to describe these bodies, especially in dilute solution, since they are composed of various HA polymer fragments and are likely to be more structurally diverse than customary micelles. ${ }^{11,12}$ It is thought that the formation of humic pseudomicelles in aqueous solution can proceed by both intramolecular and intermolecular mechanisms. ${ }^{13-15}$ In the former, a single polymer chain arranges itself in a manner that exposes its hydrophilic parts (e.g., carboxy groups) to the aqueous surroundings, while isolating its hydrophobic portion in the center of the structure. This type of "aggregation" is not characterized by a critical micelle concentration (CMC). It has been shown that the formation of humic pseudomicelles is promoted by the presence of metal ions, especially multivalent ones. ${ }^{16}$

The secondary structure of aqueous HAs can be studied by a variety of methods. Non-intrusive procedures are preferred, since the arrangements of the polymers in water are both changeable and fragile, precluding techniques that interfere with the integrity of the system. Studies of this kind are of interest because of the ubiquitous nature of humic substances in the environment, and their implication in the transport of pollutants through soil. ${ }^{17-21}$
In view of the variations among HAs from different origins, it is important to compare their behavior in aqueous solution, in order to understand how their environmental interactions may differ. The present communication offers a comparison of 6 HAs, studied by 5 different methods.

\section{Experimental}

Humic acids and reagents

Latahco silt loam humic acid (LSLHA) was obtained from the top $30 \mathrm{~cm}$ of a Latahco silt loam soil (Argiaquic Xeric Argialbolls), maintained as pasture for at least 20 years. The soil, which contained in addition to silt, $4.15 \%$ organic C, $15.9 \%$ clay, $12.1 \%$ sand, and $0.39 \%$ total $\mathrm{N}(\mathrm{w} / \mathrm{w})$, was air dried and crushed to pass a $2.0 \mathrm{~mm}$ sieve. HA extraction was carried out according to the standard procedure published by the International Humic Substances Society. ${ }^{22}$ Ritzville soil humic acid (RSHA) was isolated in the same way from a Ritzville silt loam, obtained from Pacific Northwest National Laboratories (Richland, WA). This soil contained (w/w) 43.3\% sand, 43.6\% silt, $12.3 \%$ clay, and $0.7 \%$ organic C. Pilayella littoralis humic acid (PLVHA) was obtained from the Barnett Institute (Northeastern University, Boston, MA), where it was isolated from a brown alga by a procedure described by Ghabbour et $a l .{ }^{23}$ The other HAs used were reference materials obtained from the International Humic Substances Society (St. Paul, $\mathrm{MN}$ ) and are described in the product literature.

Various characteristics of the 6 HAs used in this study have been discussed previously. ${ }^{3,10-12,15}$ Relevant information on these materials, in addition to the isolation procedures reported above, includes:

LSLHA: this is a soil HA, considered to be comprised of flexible polymers containing numerous saturated hydrocarbon links.

LHA: this HA is found in association with lignite deposits and is mined at various sites in North America. It is a condensed aromatic material with little molecular flexibility.

SRHA: this is an aquatic HA with a relatively high functional group density. In this respect it resembles a large fulvic acid.

SHHA: this IHSS standard has a high aliphatic and heteroaliphatic content.

RSHA: this material was obtained from a sandy Ritzville soil $(0.7 \%$ organic carbon). Little is known about its structure, although it appears to have a relatively high aromatic content. 
PLVHA: this new algal HA was obtained from Pilayella littoralis by the Davies group at Northeastern University, Boston. The typical elemental composition was: ${ }^{23} 42.6 \% \mathrm{C}$, $5.2 \% \mathrm{H}, 5.2 \% \mathrm{~N}, 0.8 \% \mathrm{~S}, 38.5 \% \mathrm{O}$, and $6.1 \%$ ash. IR, UV, and PMR spectra showed the material to be similar to compost HA.

Pyrene $(98 \%)$ was obtained from Sigma and purified by recrystallization from absolute ethanol and sublimation onto a cold finger. $\mathrm{MgCl}_{2}$ (J.T. Baker) and aqueous ammonia (Fisher) were used as received. Tris buffer [tris(hydroxymethyl)aminomethane] was obtained from Sigma and prepared as recommended by Cameron et al. ${ }^{24}$ All water used was treated with a $0.22 \mu \mathrm{m}$ Millipore system to $18 \mathrm{M} \Omega \mathrm{cm}$ resistivity.

\section{Procedures and instrumentation}

HA solutions were prepared by placing the appropriate amount of solid in $c a .5 \mathrm{~mL}$ of water, dropwise adding conc. aqueous ammonia until dissolution was complete (one drop was generally sufficient), and then diluting with water as needed. The solution was sonicated at $c a .40{ }^{\circ} \mathrm{C}$ until no more ammonia odor could be detected. The final $\mathrm{pH}$ of the solutions was in the range 6.5-7.0. For pyrene containing HA solutions, a $1.0 \times 10^{-7} \mathrm{M}$ aqueous pyrene solution was used as the diluent. The addition of ammonia had no effect on the humic solutes other than expediting their dissolution.

Fluorescence intensity measurements were taken with a Hitachi F-4500 fluorescence spectrophotometer. Data reported as a function of $\mathrm{Mg}^{2+}$ concentration were obtained by adding successive microliter volumes of a $0.1 \mathrm{M} \mathrm{MgCl}_{2}$ solution to $3 \mathrm{~mL}$ of HA solution. Dilution effects were thus negligible. Fluorescence intensities were measured exactly $10 \mathrm{~min}$ after each salt addition. Pyrene fluorescence was excited at $240 \mathrm{~nm}$ (where HAs are only weakly excited) and measured at $372 \mathrm{~nm}$.

SEC separations were carried out on a SigmaChrom GFC1300 gel filtration column $(13.25 \mathrm{~mL} ; 300 \times 7.5 \mathrm{~mm})$, supplied by Supelco (Bellefonte, PA). This contains a crosslinked polysaccharide packing which can tolerate eluent $\mathrm{pH}$ values in the range 3-12. Cameron et al. ${ }^{24}$ noted that used with a Tris buffer, this type of stationary phase minimizes gel-solute interactions other than size exclusion. A Waters (Milford, MA) 510 HPLC pump operating at a back pressure of $<250 \mathrm{psi}$, and a Waters 411 absorbance detector set at $280 \mathrm{~nm}$ were used. The eluent was a $1 \mathrm{M}, \mathrm{pH} 9$, Tris buffer, and the flow rate was $0.5 \mathrm{~mL} \mathrm{~min}^{-1}$. HA solutions of $50 \mathrm{ppm}$ in Tris were introduced with a $10 \mu \mathrm{L}$ injection loop. Before each run, the column was eluted with Tris buffer for $c a .1 \mathrm{~h}$, and after completion of all runs it was flushed and stored with $20 \%$ ethanol. The void volume of the system was $c a .3 \mathrm{~mL}$.

Cloud point (CP) determinations were made visually on 50 or $100 \mathrm{ppm}$ HA solutions. The ionic strength requirements were determined by heating the solution to $70^{\circ} \mathrm{C}$ (above the $\mathrm{CP}$ in all cases) and maintaining this temperature. The warm solution was titrated with $0.1 \mathrm{M} \mathrm{MgCl}_{2}$ (with temperature equilibration after each addition) until clouding was first observed. The $\mathrm{Mg}^{2+}$ concentration at this point was taken as the minimum needed. The solution was then cooled, with sonication, until the clouding disappeared, and was reheated at a rate of $1-2$ deg $\min ^{-1}$ until it reappeared. The temperature at this point was recorded as the CP.

The pyrene $I_{1} / I_{3}$ ratios were measured with a SLM Aminco 8100 fluorescence spectrophotometer. The values were determined by taking the HA/pyrene emission spectrum at $240 \mathrm{~nm}$ excitation, subtracting the HA-blank emission at this excitation (which was minimal), and taking the ratio of the emission peaks at $372 \mathrm{~nm}\left(I_{1}\right)$ and $383 \mathrm{~nm}\left(I_{3}\right)$. For measurements involving $\mathrm{Mg}^{2+}$, successive aliquots of a $\mathrm{MgCl}_{2}$ solution in the microliter range (causing negligible dilution) were added. Measurements were taken 10 min after each addition.

For the measurement of the HA fluorescence anisotropy, the
T-optics arrangement of the SLM Aminco fluorimeter was employed. The instrument has two emission channels, $180^{\circ}$ apart, each comprising a Glan-Taylor polarizer. By adjusting one to pass $I_{\|}$and the other to pass $I_{\perp}$ (for an explanation of the process, vide infra), the anisotropy could be determined in a single measurement. The reported values, recorded at $340 \mathrm{~nm}$ excitation and $400 \mathrm{~nm}$ emission, were an average of 40 determinations.

\section{Results and discussion}

\section{HPSEC}

The size exclusion chromatograms of the 6 HAs under investigation are shown in Fig. 1. The variable small peak that emerged at $3-4 \mathrm{~mL}$ of eluent in five of the samples represents the large components that were totally excluded by the gel filtration medium. This corresponded to a globular protein exclusion limit of $1.3 \times 10^{6} \mathrm{Da}$, and a blue dextran limit of $>2.0 \times 10^{6} \mathrm{Da}$. No unequivocal HA size assignments can be made on the basis of these compounds, but the totally excluded humic polymers should be expected to have molecular weights in the $100+\mathrm{kDa}$ range. The main peak in all chromatograms represents a continuous distribution of molecular sizes. The absence of multiple peaks within this distribution indicates that no interactions other than size exclusion were likely to play a role in the chromatographic process. It can be seen that while the molecular sizes of the six HAs covered similar ranges, definite differences existed in their distributions. As would be expected for an aquatic HA, SRHA lacked the large components present in the other samples. Also, its distribution was notably narrow, possibly suggesting a degree of size selectivity for SRHA dissolution in river water. The chromatograms of LSLHA and SHHA are clearly similar, while that of RSHA shows a greater abundance of large components. This is not only indicated by the size of the totally excluded peak, but also by the relatively high level of the region immediately

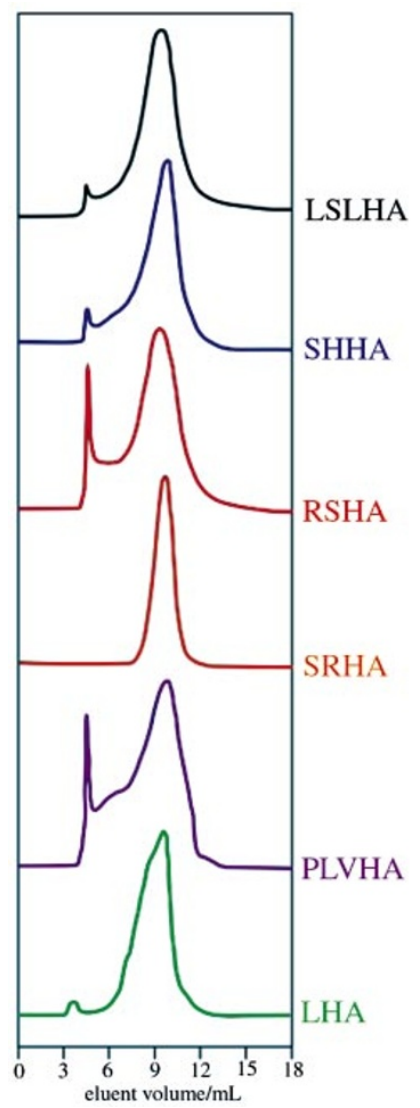

Fig. 1 Size exclusion chromatograms of HAs. 
following this peak, which represents the large retained components of the sample. A similar situation was found for the algal material, PLVHA, which also shows a broad distribution. In contrast, LHA had a modest totally excluded peak and a relatively small complement of large polymers.

\section{Fluorescence enhancement}

It has been shown in previous studies ${ }^{11,13,16}$ that the fluorescence intensity of a pyrene probe present in an aqueous HA solution is in many cases intensified when metal ions are added. This phenomenon was ascribed to the enhancement of pseudomicellar domains in HA by the cations. The sequestration of pyrene in these structures is thought to lead to a reduction in its quenching encounters with hydrophilic HA moieties. It has been proposed that the degree to which a HA responds to the addition of a metal in this manner is (at least partially) a function of its molecular flexibility. Fig. 2 shows the change of pyrene fluorescence with the concentration of added $\mathrm{Mg}^{2+}$ for solutions of the HAs under investigation. It is clear that LSLHA and RSHA produced strong fluorescence enhancement, while SHHA, LHA, and SRHA did so rather weakly. In the context of the micellar model, this means that the first two HAs formed pseudomicelles most effectively upon the addition of the cation, providing the most suitable sequestration sites for pyrene. Interestingly, PLVHA produced a decrease, rather than increase, in pyrene fluorescence when $\mathrm{Mg}^{2+}$ was added. This suggests that enhanced pseudomicellization did not take place in this case, but that the fluorophore underwent increased exposure to $\mathrm{Cl}^{-}$ions (weak quenchers of pyrene fluorescence). In the other HA solutions, the chloride ions (being hydrophilic) were excluded from the humic pseudomicelles. There is no evidence of a useful correlation between the fluorescence enhancement phenomenon and the molecular size distributions shown in Fig. 1.

\section{$I_{1} / I_{3}$ ratio}

The intensity ratio of the first and the third vibronic peaks in the emission spectrum of pyrene has been shown to constitute a good measure of the polarity of the environment of the molecule. ${ }^{25}$ The $I_{1} / I_{3}$ ratio is high in polar surroundings (e.g., 1.8 in water) and low (0.9-1.3) in nonpolar solvents. The parameter was employed in a previous $\mathrm{HA} /$ pyrene study, where it was found to be useful, albeit of limited sensitivity. ${ }^{15}$ In the present case, its variation with $\mathrm{Mg}^{2+}$ concentration in $10 \mathrm{ppm}$ solutions of HA was determined (Fig. 3). Here, too, the range over which the values changed was fairly small, but definite trends could be observed in at least two cases. For pyrene in LSLHA and RSHA solutions, $I_{1} / I_{3}$ decreased sharply as $\mathrm{Mg}^{2+}$ was added, indicating that the probe moved to less polar surroundings. This is in keeping with the proposed sequestration mechanism, i.e. the formation of enhanced pseudomicellar domains through interactions with the cation. In the SHHA

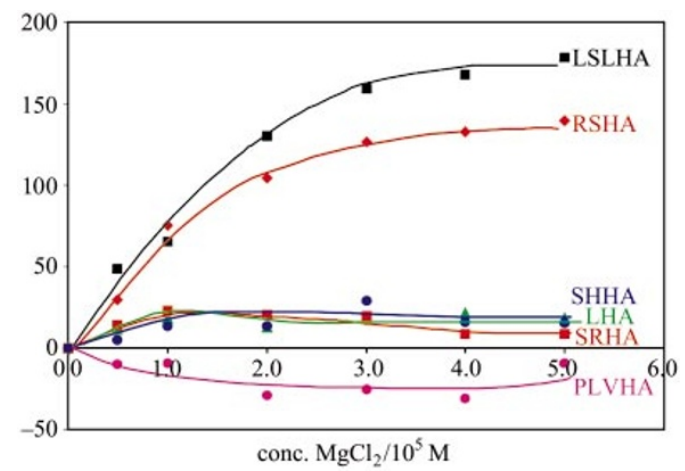

Fig. 2 Variation of the fluorescence intensity of $1.0 \times 10^{-7} \mathrm{M}$ pyrene with $\mathrm{Mg}^{2+}$ concentration in 10 ppm HA solutions.

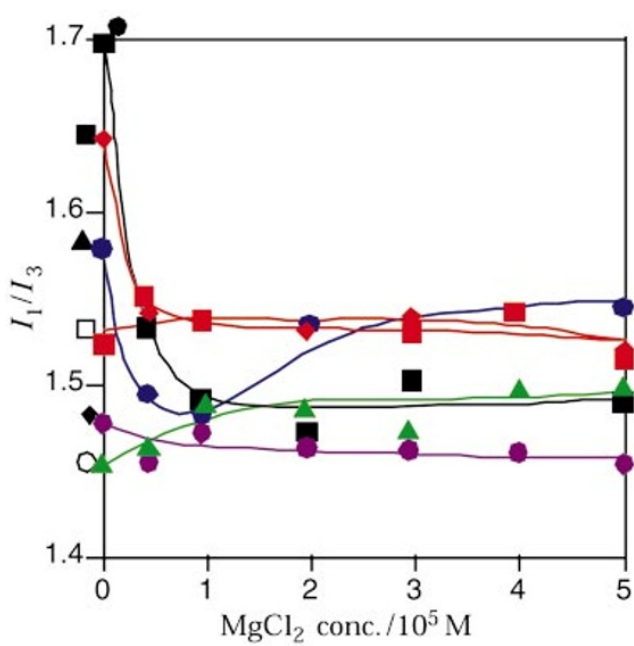

Fig. 3 Variation of the $I_{1} / I_{3}$ ratio of the pyrene $\left(1.0 \times 10^{-7} \mathrm{M}\right)$ emission with $\mathrm{Mg}^{2+}$ concentration in 10 ppm HA solutions. LSLHA (ם); RSHA $(\downarrow)$; SRHA $(\boldsymbol{\square})$; SHHA $(\bullet)$; $\operatorname{PLVHA}(\bullet)$; LHA $(\pi)$.

solution, an initial decrease in $I_{1} / I_{3}$ was observed, but the ratio increased again when more $\mathrm{Mg}^{2+}$ was added. The reason for the rise at the higher cation concentrations is not immediately clear, but suggests that pseudomicelle formation reached a plateau at a $\mathrm{Mg}^{2+}$ concentration of $c a .1 \times 10^{-5} \mathrm{M}$. Addition beyond this concentration exposed the fluorophore to a microenvironment of higher ionic strength. The solutions of PLVHA, SRHA, and LHA produced no discernible trends, suggesting that cation induced pseudomicelle formation/ enhancement was less effective in these HAs. For the case of LHA this has been noted before ${ }^{11}$ and was ascribed to the condensed aromatic nature of this material. This was considered to limit its flexibility and hence its ability to undergo effective intramolecular rearrangements upon the addition of cations. This is also likely to be the case for SRHA, being a relatively small, aquatic material. PLVHA, the newly discovered HA, appears to have relatively little tendency to undergo cation induced rearrangements, leading to the formation of enhanced hydrophobic domains. It should be noted that the $I_{1} / I_{3}$ data are in good agreement with the results of the fluorescence enhancement study.

\section{Cloud points}

The clouding phenomenon of amphiphilic solutes in water is best known for nonionic surfactants, especially those with polyoxyethylene (POE) head groups. ${ }^{26}$ It refers to a spontaneous phase separations in micellar solutions of these compounds when the temperature is raised to a value known as the cloud point $(\mathrm{CP}$; a characteristic parameter of the surfactant). Clouding is due to the decrease of the dielectric constant of water with temperature, which diminishes its $\mathrm{H}$ bonding with the POE chains, causing their dehydration. In keeping with this, ionic surfactants do not cloud. It has been shown ${ }^{13}$ that HA solutions also undergo clouding, provided the ionic strength of the solution is high enough. This requirement is ascribed to the fact that HAs are, to a variable degree, anionic in aqueous solution and will not dehydrate unless the charges are sufficiently neutralized.

Table 1 shows the CPs and relevant parameters of the HAs under investigation. Attempts were made to use $50 \mathrm{ppm}$ HA concentrations in all cases, but with SHHA and SRHA this proved insufficient to produce visible clouding. LSLHA required the lowest cation concentration and clouded at the lowest temperature. This indicates that this HA most easily eliminated water from its hydrated parts, and also suggests that its chains possessed the necessary flexibility to contract and form hydrophobic structures. With LHA and RSHA, both the 
Table 1 Clouding characteristics of HA solutions

\begin{tabular}{|c|c|c|c|}
\hline HA & {$[\mathrm{HA}](\mathrm{ppm})$} & Min. $\mathrm{MgCl}_{2}$ conc. $/ 10^{3} \mathrm{M}$ & $\mathrm{CP} /{ }^{\circ} \mathrm{C}$ \\
\hline LSLHA & 50 & 1.4 & 41 \\
\hline LHA & 50 & 2.9 & 47 \\
\hline RSHA & 50 & 3.8 & 55 \\
\hline PLVHA & 50 & 3.5 & 62 \\
\hline SHHA & 100 & 15 & 58 \\
\hline SRHA & 100 & 28 & 64 \\
\hline
\end{tabular}

minimum $\mathrm{Mg}^{2+}$ concentration and the $\mathrm{CP}$ increased, while the $\mathrm{CP}$ was elevated still further in PLVHA. This indicates that these HAs underwent hydrophobic aggregation with increasing difficulty, which may be ascribed to less molecular flexibility and/or stronger H-bonding. SHHA and SRHA required higher sample concentrations, and commensurately higher $\mathrm{Mg}^{2+}$ concentrations, and clouded at relatively high temperatures. Even with the HA concentrations normalized to $50 \mathrm{ppm}$, however, the ionic strength requirements were high in these cases. This is especially true for SRHA, which may be attributed to the relatively small size (Fig. 1) and high water compatibility of this aquatic HA.

\section{Fluorescence anisotropy}

In this technique, both the exciting radiation and the emission used in the fluorescence measurement are passed through polarizers. $^{27}$ The static fluorescence anisotropy, $r$, of a fluorophore is defined in eqn. (1):

$$
r \quad \begin{array}{cc}
I_{\|} & I_{\perp} \\
\hline I_{\|} & 2 I_{\perp}
\end{array}
$$

where $I_{\|}$is the emission intensity measured with the emission polarizer aligned parallel with the excitation polarizer, and $I_{\perp}$ is the intensity obtained with the emission polarizer turned $90^{\circ}$ The value of $r$, which can attain a maximum of 0.4 , is a measure of the rotational diffusion of the fluorophore in solution. Species that diffuse slowly, either due to their molecular size or to solvent interactions (e.g., H-bonding) generally have higher anisotropies than those diffusing quickly.

In aqueous solution at room temperature, dilute HA solutions have anisotropies close to zero, revealing little about differences in their mobilities. In the present case, therefore, the HAs were dissolved in glycerol, which, owing to its high viscosity, gives rise to more substantial anisotropy values. Glycerol interactions with HA are clearly different from those of water, but meaningful relative mobility data can still be obtained in this solvent. Fig. 4 shows the anisotropy values

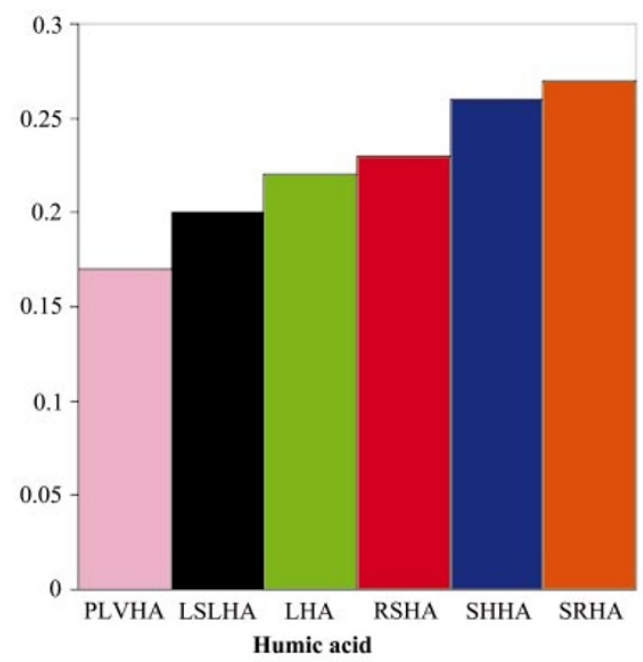

Fig. 4 Fluorescence anisotropy values of 5 ppm HA solutions in glycerol at room temperature. of dilute HA solutions in glycerol. It can be seen that SRHA is the least mobile, and PLVHA the most. The data do not $a$ priori reveal whether the mobility differences shown were due to the sizes of the HAs, their H-bonding to the solvent, or a combination of these. However, when taken together with the information in Fig. 1, which shows that PLVHA was among the largest of the HAs and LSLHA and SHHA were similar in size distribution, it is reasonable to surmise that solvent interactions were preeminent in determining the rotational diffusion of these solutes. In view of the known hydrophilic nature of SRHA, it is understandable that this HA interacted strongly with an H-bonding solvent such as glycerol. The other HAs were solvated in decreasing order of anisotropy as shown in Fig. 4.

\section{Conclusions}

Conclusions drawn from the measurements discussed above are by necessity tentative, since the results are without exception open to interpretation. It has been noted that the fluorescence enhancement and $I_{1} / I_{3}$ results are in good agreement, indicating that LSLHA and RSHA form pseudomicelles easily under the influence of cations in solution. SHHA, LHA and SRHA do so to a lesser degree, while PLVHA does not. The CP behavior suggests that LSLHA also aggregates most easily on a macroscopic scale, while its anisotropy indicates that it is not as thoroughly solvated (at least by glycerol) as some of the other HAs. Cautious extension of this observation to other H-bonding media suggests that the behavior of LSLHA in water is related to its lack of strong solvent interactions. The high $\mathrm{CP}$ and high anisotropy of SHHA indicate that this HA is well solvated. LHA has a fairly low $\mathrm{CP}$, showing that macroscopic aggregation (the formation of particles that scatter visible light) proceeds more easily than pseudomicellization. This could mean that association is largely intermolecular in this case, which fits the known condensed aromatic character of LHA. The findings on SRHA are most unequivocal among those for the HAs studied: low fluorescence enhancement, low $I_{1} / I_{3}$, high $\mathrm{CP}$, and high anisotropy all indicate that this aquatic HA is well solvated and has little tendency to aggregate - both in the pseudomicellar and the macroscopic sense. An interesting case is presented by PLVHA, which, being a recently discovered material, has not been extensively studied. The SEC data show a broad size distribution, and both fluorescence enhancement and $I_{1} / I_{3}$ could mean that minimal pseudomicelle formation takes place. The small anisotropy value suggests relatively low solvation, while the high CP indicates the opposite. This discrepancy may in this instance be related to the different solvents used for the two measurements.

\section{Acknowledgement}

The authors thank the EPA (R82-2832-010) for financial support.

\section{References}

1 R. S. Swift, Macromolecular properties of soil humic substances: fact, fiction, and opinion, Soil Sci., 1999, 164, 790.

2 K. A. Thorn, D. W. Folan and P. MacCarthyn, Characterization of the International Humic Substances Society Standard and Reference Fulvic and Humic Acids by Solution State Carbon-13 and Hydrogen-1 Nuclear Magnetic Resonance Spectrometry, Water Resources Investigations Report 89-4196, US Geological Survey, Denver, CO, 1989.

3 M. A. Anderson, A. Hung, D. Mills and M. S. Scott, Factors affecting the surface tension of soil solutions and solutions of humic acids, Soil Sci., 1995, 160, 111.

4 C. T. Chiou, R. L. Malcolm, T. I. Brinton and D. E. Kile, Water solubility enhancement of some organic pollutants and pesticides 
by dissolved humic and fulvic acids, Environ. Sci. Technol., 1986, 20, 502 .

5 R. A. Larson and S. A. Rounds, Photochemistry in Aqueous Surface Layers, in Photochemistry in Environmental Aquatic Systems, ed. R. G. Zika and W. J. Cooper, American Chemical Society, Washington, DC, 1987.

6 D. E. Kile and C. T. Chiou, Water-Solubility Enhancement of Nonionic Organic Contaminants, in Aquatic Humic Substances, ed. I. H. Suffet and P. MacCarthy, American Chemical Society, Washington, DC, 1989.

7 W. Rochus and S. Sipos, Micelle formation by humic substances, Agrochimica, 1978, 22, 446.

8 R. L. Wershaw, A new model for humic materials and their interactions with hydrophobic organic chemicals in soil-water or sediment-water systems, J. Contam. Hydrol., 1986, 1, 29.

9 R. L. Wershaw, Membrane-micelle model for humus in soils and sediments and its relation to humification, Open file report 91-513, US Geological Survey, Denver, CO, 1992.

10 R. L. Wershaw, Environ. Sci. Technol., 1993, 27, 814.

11 R. R. Engebretson and R. von Wandruszka, Microorganization in dissolved humic acids, Environ. Sci. Technol., 1994, 28,1934.

12 R. R. Engebretson and R. von Wandruszka, Kinetic aspects of cation-enhanced aggregation in aqueous humic acids, Environ. Sci. Technol., 1998, 32, 488 .

13 R. von Wandruszka, The micellar model of humic acid: evidence from pyrene fluorescence measurements, Soil Sci., 1998, 163, 921.

14 L. M. Yates, R. R. Engebretson, T. M. Haakenson and R. von Wandruszka, Immobilization of aqueous pyrene by dissolved humic acid, Anal. Chim. Acta, 1997, 356, 295.

15 R. R. Engebretson and R. von Wandruszka, The effect of molecular size on humic acid associations, Org. Geochem., 1997, 26, 759 .

16 R. von Wandruszka, C. Ragle and R. R. Engebretson, The role of selected cations in the formation of pseudomicelles in aqueous humic acid, Talanta, 1997, 44, 805.
17 E. M. Murphy, J. M. Zachara and S. C. Smith, Influence of mineral-bound humic substances on the sorption of hydrophobic organic compounds, Environ. Sci. Technol., 1990, 24, 1507.

18 T. T. Ames and E. A. Grulke, Group contribution method for predicting equilibria of nonionic organic compounds between soil organic matter and water, Environ. Sci. Technol., 1995, 29, 2273.

19 Y. O. Aochi and W. J. Farmer, Role of microstructural properties in the time-dependent sorption/desorption behavior of 1,2dichloroethane on humic substances, Environ. Sci. Technol., 1997, 31, 2420

20 G. M. Litton and G. L. Guymon, Laboratory experiments evaluating the transport and fate of DBCP in Hanford sandy loam, J. Environ. Qual., 1993, 22, 311.

21 B. R. Magee, L. W. Lion and A. T. Lemley, Transport of dissolved organic macromolecules and their effect on the transport of phenanthrene in porous media, Environ. Sci. Technol., 1991, 25, 323.

22 Product literature published on January 25, International Humic Substances Society, St. Paul, MN, 1985.

23 E. A. Ghabbour, A. H. Khairy, D. P. Cheney, V. Gross, G. Davies, T. R. Gilbert and X. Zhang, Isolation of humic acid from the brown alga Pilayella littoralis, J. Appl. Phycol., 1994, 6 459

24 R. S. Cameron, R. S. Swift, B. K. Thornton and A. M. Posner, Calibration of gel permeation chromatography materials for use with humic acid, J. Soil Sci., 1972, 23, 342.

25 D. C. Dong, M. A. Winnik, The py scale of solvent polarities. Solvent effects on the vibronic fine structure of pyrene fluorescence and empirical correlations with ET and Y values, Photochem Photobiol., 1982, 35, 1721.

26 W. L. Hinze and E. Pramauro, A critical review of surfactantmediated phase separation (cloud point extraction): theory and applications, Crit. Rev. Anal. Chem., 1993, 24, 133.

27 J. R. Lackowicz, Principles of Fluorescence Spectroscopy, Kluwer Academic, New York, 2nd edn., 1999, pp. 291-316. 